\title{
Highly efficient multiuser detector of M-PSK signals using coordinate-descent phase estimation
}

\author{
Yuriy Zakharov ${ }^{\dagger}$, Senior Member, IEEE, Zhi Quan ${ }^{\ddagger}$, George White and Junruo Zhang $^{\dagger}$ \\ ${ }^{\dagger}$ Department of Electronics, University of York, UK, e-mail: (yz1,jz519)@ohm.york.ac.uk. \\ $\ddagger$ Department of Electrical Engineering, Federal University of Juiz de Fora, MG, Brazil, e-mail: zhi.quan@engenharia.ufj.br. \\ \# QinetiQ Ltd., Malvern, Worcestershire, UK, e-mail: gpwhite@qinetiq.com.
}

\begin{abstract}
Joint detection of a large number of M-PSK symbols is important for many applications in communications, in particular, for multiuser detection in CDMA systems. In the past, there was much effort to find a technically simple solution to this problem and yet providing near optimal detection performance. We propose to use a novel iterative technique, the phase descent search (PDS) algorithm, for solving this problem. This technique constrains the solution to have a unit magnitude and it is based on coordinate descent iterations where coordinates are the unknown symbol phases. The PDS algorithm, together with a descent local search (also implemented as a version of the PDS algorithm), is used multiple times with different initializations in a proposed multiple phase detector; the solution with the minimum cost is then chosen as the final solution. Simulation results show that for highly loaded multiuser scenarios the proposed technique has a detection performance that is close to the single-user bound. The results also show that the multiple phase detector allows detection in highly overloaded scenarios and it exhibits near-far resistance. In particular, the detector has a performance that is significantly better, and complexity that is significantly lower, than that of the detector based on semi-definite relaxation.
\end{abstract}

Index Terms - coordinate descent, low complexity, M-PSK, multiuser detection, quadratic optimization, semi-definite relaxation

\section{INTRODUCTION}

In multiple-access CDMA systems, multiuser detection is capable of providing high detection performance [1]. However, the complexity of multiuser detectors that are capable of approaching the optimal performance is still a very important issue. For a small-size problem, sphere decoding achieves a nearly optimal performance [2], but becomes complicated when the size of the problem increases [3]. Semi-definite relaxation (SDR) has also been proposed and investigated for joint detection of a number of symbols with M-PSK modulation [4]. Although, promising for multiuser detection, SDR is still complicated for practical implementation [5]. An efficient implementation of multiuser detection, especially in hardware, can be based on the dichotomous coordinate descent (DCD) algorithm [6], [7], [8]. However, the box-constrained detector does not show good detection performance in highly loaded scenarios. In [9], we proposed a novel technique for solving a constrained optimization problem corresponding to the optimal detection of M-PSK signals in MIMO systems. This technique has the detection performance similar to that of the sphere decoder and significantly lower complexity. Later, we have found that this new technique is especially efficient for scenarios where the number of unknown symbols is large. This is typical for CDMA communications. In this paper, we consider joint detection of M-PSK symbols in application to multiuser CDMA scenarios and propose to use the technique from [9] for this purpose. We show that such a detector has a detection performance that is better than that of the SDR detector and significantly lower complexity.

\section{Problem Formulation}

The matched-filter output of a symbol synchronous CDMA receiver is given by the $K$-length vector

$$
\mathbf{y}=\mathbf{R} \mathbf{b}+\mathbf{n}
$$

where the vector $\mathbf{b} \in \mathcal{A}^{K}=\left\{\left[e^{j 2 \pi m / M}\right]_{m=0}^{M-1}\right\}^{K}$ contains MPSK symbols (from a constellation set $\mathcal{A}$ ) transmitted by the $K$ users, $\mathbf{R}$ is a $K \times K$ positive definite signature waveform correlation matrix, and $\mathbf{n}$ is a complex-valued zero-mean Gaussian random vector with covariance matrix $\sigma^{2} \mathbf{R}, \sigma^{2}>0$ [5].

The maximum likelihood (ML) multiuser detector estimates the vector $\mathbf{b}$ by minimizing the quadratic function [1]

$$
J(\mathbf{b})=\mathbf{b}^{H} \mathbf{R} \mathbf{b}-2 \Re\left\{\mathbf{y}^{H} \mathbf{b}\right\}
$$

with the constraint $\mathbf{b} \in \mathcal{A}^{K}$, where $\Re\{\cdot\}$ denotes the real part of a complex number. The ML data estimates are given by

$$
\hat{\mathbf{b}}_{\mathrm{ML}}=\arg \min _{\mathbf{b} \in \mathcal{A}^{K}}\{J(\mathbf{b})\} .
$$

Although the ML detector provides the best detection performance, it is not practical due to its high complexity [1]. Relaxation of the constraint is a general approach that results in lower complexity multiuser detectors [5]. As M-PSK symbols have a unit magnitude, we use the following relaxation to the original problem (3):

$$
\hat{\mathbf{b}}=\arg \min _{\left|b_{k}\right|=1, k=0, \ldots, K-1}\{J(\mathbf{b})\},
$$

where $b_{k}$ are elements of the vector $\mathbf{b}$. After obtaining an approximate solution to this problem, the final data estimates are obtained by mapping elements of the solution vector into the constellation set $\mathcal{A}$.

Notice that the problem (4) is non-convex. Using a convex optimization technique, like the coordinate descent method 
TABLE I

Phase DesCent Search algorithm

\begin{tabular}{|c|c|c|c|}
\hline Step & Equation & $x$ & + \\
\hline Init. & $\mathbf{b}=\mathbf{b}_{0}, \boldsymbol{\phi}=\phi_{0}, \mathbf{r}=\mathbf{y}-\mathbf{R} \mathbf{b}_{0}, d=d_{0}, n=0$ & & \\
\hline 1 & for $m=1: M_{b}$ & & \\
\hline 2 & $d \leftarrow \lambda d$ & - & - \\
\hline 3 & $\boldsymbol{\theta}=\operatorname{diag}\{\mathbf{R}\}[1-\cos (d)]$ & $K$ & - \\
\hline 4 & Flag $=0$ & - & - \\
\hline 5 & for $p=0:(K-1)$ (a pass) & - & - \\
\hline 6 & $\phi_{p, 1}=\phi_{p}+d, b_{p, 1}=e^{j \phi_{p, 1}}$ & - & 1 \\
\hline 7 & $\Delta_{1}=b_{p, 1}-b_{p}, T_{1}=\Re\left\{\Delta_{1}^{*} r_{p}\right\}$ & 2 & 2 \\
\hline 8 & if $\theta_{p}<T_{1}$ & - & 1 \\
\hline 9 & $n \leftarrow n+1$, Flag $=1$ & - & - \\
\hline 10 & $\mathbf{r} \leftarrow \mathbf{r}-\Delta_{1} \mathbf{R}^{(p)}$ & $4 K$ & $4 K$ \\
\hline 11 & $\phi_{p}=\phi_{p, 1}, b_{p}=b_{p, 1}$ & - & - \\
\hline 12 & $\phi_{p, 2}=\phi_{p}-d, b_{p, 2}=e^{j \phi_{p, 2}}$ & - & 1 \\
\hline 13 & $\Delta_{2}=b_{p, 2}-b_{p}, T_{2}=\Re\left\{\Delta_{2}^{*} r_{p}\right\}$ & 2 & 2 \\
\hline 14 & if $\theta_{p}<T_{2}$ & - & 1 \\
\hline 15 & $n \leftarrow n+1$, Flag $=1$ & - & - \\
\hline 16 & $\mathbf{r} \leftarrow \mathbf{r}-\Delta_{2} \mathbf{R}^{(p)}$ & $4 K$ & $4 K$ \\
\hline 17 & $\phi_{p}=\phi_{p, 2}, b_{p}=b_{p, 2}$ & - & - \\
\hline 18 & end the loop over $p$ (end of the pass) & - & - \\
\hline 19 & if $n>N_{u}$ the algorithm stops & - & - \\
\hline 20 & if Flag $=1$ go to step 4 & - & - \\
\hline 21 & end the loop over $m$ & - & - \\
\hline & Total complexity: $\leq$ & IIC & \\
\hline
\end{tabular}

considered below, can result in a local minimum. Multiple solutions of the problem for a set of initializations will allow finding a 'smallest' local minimum. Nevertheless, as will be seen below, a small number of such initializations will result in a very high performance of the detector.

\section{PROPOSED DETECTOR}

The multiuser detector is based on multiple use of the Phase Descent Search (PDS) algorithm [9] as described below.

\section{A. Phase Descent Search (PDS) algorithm}

The PDS is based on coordinate descent iterations with respect to the unknown symbol phases and a constraint that forces the symbols to have a unit magnitude. Specifically, the elements of the solution are given by

$$
b_{k}=e^{j \phi_{k}}, k=0, \ldots, K-1, \phi_{k} \in[-\pi, \pi] .
$$

The coordinate descent iterations are applied to the phases $\phi_{k}$. The derivation of the PDS algorithm is based on a general coordinate descent method as described in [10] (see also [6]). To derive the PDS algorithm, we apply the coordinate descent method to the cost function (2) with elements $b_{k}$ from (5); see [9] for details of the derivation. The iterative PDS algorithm is summarized as shown in Table $\mathrm{I}$.

The complex valued solution vector $\mathbf{b}$ is represented using real and imaginary parts; its entries are initialized according to (5), i.e., $\left|\left[\mathbf{b}_{0}\right]_{k}\right|=1$ for all $k=1, \ldots, K$. The phases of the entries are also saved in a $K$-length vector $\phi$. The algorithm has two loops. In the external loop, $m=1: M_{b}$, the step-size parameter $d$, defining the resolution of the phase estimation, is reduced $M_{b}$ times according to the relationship $d \leftarrow \lambda d$, i.e., $d=d_{0} \lambda^{m}, 0<\lambda<1$. The initial value of the stepsize parameter is $d_{0}=2 \pi$. The choice of $\lambda$ may relate to the modulation scheme used; this will be addressed below. The parameter $M_{b}$ indicates the number of reductions of the stepsize $d$ and, thus, the final phase resolution $d_{0} \lambda^{M_{b}}$; e.g., in the case of $\lambda=1 / 2$ and $M_{b}=6$, the final phase resolution is $2 \pi / 2^{M_{b}}=\pi / 32$.

In the internal loop, $p=0:(K-1)$, all elements of the solution vector are checked for a possible update. If, for the $p$ th element, one of the inequalities at steps 8 or 14 is satisfied, the iteration is called 'successful' and the element $b_{p}$ of the solution vector and the residual vector $\mathbf{r}$ are updated (steps 10-11 or 1617, respectively); otherwise, they are not changed. The binary parameter Flag indicates a successful iteration. The variable $n$ counts the number of successful iterations; it is used for introducing the stopping criterion at step 19, where $N_{u}$ is a predefined parameter that limits the maximum number of updates. If for a pass through all $K$ solution elements in the internal loop, there is no update, it means that, for the current solution, the step-size $d$ is too large and it requires reduction. The step-size is reduced at step 2. Thus, further updates of the solution will be done with a higher phase resolution.

\section{B. Multiple Phase Detector}

The PDS algorithm with a consequent mapping of the solution to the constellation $\mathcal{A}$ will be shown to provide a good detection performance for BPSK signals, i.e., in the case $M=2$. However, for M-PSK signals with $M>2$, it does not guarantee high performance. This is because, for a particular initialization, the PDS algorithm can find a local minimum of the cost function instead of the global minimum. The performance can be improved by multiple use of the PDS algorithm with different starting solutions and a further local search [5] (also implemented using the PDS algorithm) in the multiple phase detector as shown in Table II. The multiple use of search algorithms with different starting solutions is known to be a simple approach for improving the detection performance [5]. Moreover, this approach introduces parallelism in the processing thus making a hardware implementation of the detector efficient.

The PDS algorithm is used multiple times with different initializations of the solution vector. To obtain a solution for the $q$ th initialization $\mathbf{b}_{q}$, we apply the PDS algorithm twice. The first PDS (step 1 in Table II) with the consequent mapping (step 2) to the constellation $\mathcal{A}$ provides a first solution, whereas the second PDS (step 3) performs a descent local search moving from one ML feasible solution to another in the neighborhood of the first solution [5]. Among $Q$ solutions, the one that has the smallest cost function $J(\cdot)$ (computed at step 4) is selected. For the first PDS, we use a high $M_{b}$ and $\lambda=1 / 2$. For the second PDS, we use $M_{b}=1$ and $\lambda=1 / M$. The second PDS is initialized by the mapped solution of the previous PDS, $\tilde{\mathbf{b}}=\exp (j \tilde{\boldsymbol{\phi}})$, and, according to the values of $M_{b}$ and $\lambda$, it performs M-PSK symbol-flipping (i.e., the descent local search).

\section{IMPLEMENTATION ISSUES}

In this section, we discuss implementation of the proposed multiple phase detector in hardware. This detector possesses intrinsic parallelism. It can be implemented as $Q$ identical parallel branches, each containing two PDS blocks (corresponding 
TABLE II

Multiple Phase Detector

\begin{tabular}{|c|c|}
\hline Step & Algorithm \\
\hline \multirow{4}{*}{1} & for $q=0: Q-1$ \\
\hline & Initialize the PDS algorithm with \\
\hline & $M_{b}>1, \lambda=1 / 2, \boldsymbol{\phi}=\boldsymbol{\phi}_{q}, \mathbf{b}=\mathbf{b}_{q}, \mathbf{r}=\mathbf{y}-\mathbf{R} \mathbf{b}_{q}$ \\
\hline & Apply the PDS algorithm to obtain a solution $\mathbf{b}$ \\
\hline 2 & Map $\mathbf{b}$ into the M-PSK constellation to obtain $\tilde{\phi}$ and $\tilde{b}$ \\
\hline \multirow[t]{3}{*}{3} & Initialize the PDS algorithm with \\
\hline & $M_{b}=1, \lambda=1 / M, \boldsymbol{\phi}=\tilde{\boldsymbol{\phi}}, \mathbf{b}=\tilde{\mathbf{b}}, \mathbf{r}=\mathbf{y}-\mathbf{R} \tilde{\mathbf{b}}$ \\
\hline & Apply the PDS algorithm to obtain a solution $\mathbf{b}$ \\
\hline \multirow[t]{3}{*}{4} & Calculate the cost function for the solution $\mathbf{b}$ \\
\hline & end \\
\hline & Choose the solution with the minimum cost \\
\hline
\end{tabular}

to steps 1 and 3 in Table II), a mapping block (step 2 in Table II) and a block for computation of the cost function (step 4 in Table II).

The first PDS blocks in the branches use different initialization of the phase vector $\phi$ and, accordingly, different initialization of the solution vector $b$ and the residual vector $r$. The initial vector $\mathbf{b}_{q}$ in the $q$ th branch should satisfy the constraint (5) and therefore it can be represented as $\mathbf{b}_{q}=\exp \left(j \phi_{q}\right)$. For a fixed vector $\phi_{q}$, the initial vector $\mathbf{b}_{q}$ can be obtained using a look-up table. The size of the look-up table is as small as $2^{M_{b}}$; e.g., for $M_{b}=6$, it contains as few as 64 complex numbers. To initialize the residual vector, one has to calculate the matrixvector product $\mathbf{R b}_{q}$, which, in the general case, would require $K^{2}$ complex-valued multiplications and $K(K-1)$ complexvalued additions in each branch. This computation is significantly simplified if all elements of the phase vector $\phi_{q}$ are the same, i.e., $\mathbf{R} \mathbf{b}_{q}=c_{q} \mathbf{R} 1$, where $c_{q}$ is a complex-valued constant and 1 is a $K$-length vector of ones. In this case, the vector R1 is calculated once for all $Q$ branches and this calculation does not require multiplications. Then, for initialization of the residual vector for each branch as few as $K$ complex-valued multiplications and $K$ complex-valued additions are required. Multiple simulations with different initialization (not presented here due to lack of space) have shown that the use of the initialization $c_{q}=\exp \left(j \frac{\pi q}{Q}\right)$ provides good detection performance.

Since the PDS algorithm provides phases of the solution, the mapping at step 2 becomes a simple operation. It includes truncation (quantization) of $M_{b}$-bit elements of the phase vector $\phi_{q}$ into $\log _{2}(M)$-bit elements of the vector $\tilde{\phi}$ and the use of a lookup table of size $M$ to obtain $\tilde{\mathbf{b}}$. These two vectors are used for initialization of the second PDS block in the branch. Note that the proposed choice of the parameters $M_{b}=1$ and $\lambda=1 / M$ makes the second PDS simple for implementation and, also, it removes the need for symbol mapping after the second PDS. Our simulation results (not presented here) have shown that, in the second PDS blocks, the parameter $N_{u}$ limiting the number of updates can be significantly lower than that in the first PDS blocks.

Now, we discuss implementation of the PDS blocks. In the PDS algorithm presented in Table I, there are two stopping criteria: 1) at step 21 upon achieving a predefined phase resolution $d_{0} \lambda^{M_{b}}$; and 2) at step 19 upon performing $N_{u}$ updates. When implementing in hardware (e.g., on an FPGA platform), other stopping criteria can also be used; e.g., the PDS can stop after a predefined number of clock cycles (or execution time). Table I

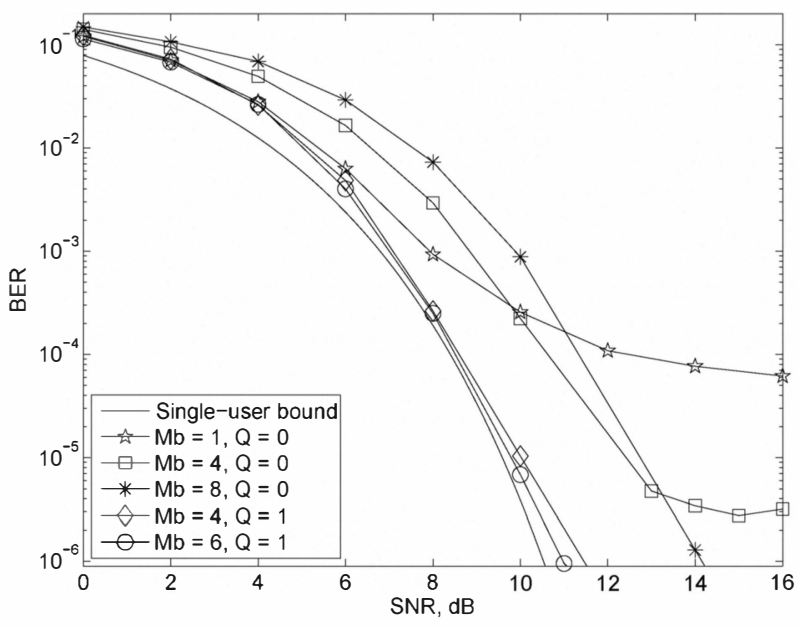

Fig. 1. BER performance of the multiple phase detector; BPSK modulation, $K=60, S F=63$.

shows the complexity of different steps of the PDS algorithm in terms of real multiplications and additions, as well as the maximum complexity. At step 3, the values $1-\cos (d)$ for $M_{b}$ values of $d$ can be precomputed. Thus, this step only requires $K$ real multiplications. For transforming phases $\phi_{p, 1}$ and $\phi_{p, 2}$ into complex numbers $b_{p, 1}$ and $b_{p, 2}$ (at steps 6 and 12, respectively), a look-up table of size $2^{M_{b}}$ can be used as explained above. The maximum complexity corresponds to a scenario where, for every pass, only one successful iteration happens and the PDS algorithm stops at step 19, i.e., due to reaching a predefined maximum number of successful updates $N_{u}$. If, in a pass through $K$ phases, there are several successful iterations and/or the PDS algorithm stops at step 21, i.e., due to reaching the predefined phase resolution, the complexity will be lower. In the simulation below, we use $N_{u}$ high enough to guarantee that the PDS algorithm stops at step 21. Therefore, our results will demonstrate the upper bound for the complexity of the detector.

\section{NUMERICAL RESULTS}

Fig. 1 shows the BER performance of the proposed detector for BPSK modulated signals against the single-user bound, obtained in $10^{6}$ simulation trials. The user signature waveforms have equal energies; they are binary and chosen randomly in each simulation trial. The number of users is $K=60$ and the spreading factor is $\mathrm{SF}=63$, i.e., here we deal with a highly loaded multiuser scenario. The case $Q=0$ corresponds to the PDS algorithm with a consequent mapping of the solution to the set $\mathcal{A}$ (steps 1 and 2 in Table II). The case $M_{b}=1$ with $\lambda=1 / 2$ corresponds to bit-flipping, i.e., changing the $p$ th coordinate of the solution vector between +1 and -1 . The bit-flipping provides a good performance at low SNRs, but, at high SNRs, there is a BER floor. As $M_{b}$ increases, the BER floor level is reduced; however, the performance at low SNRs becomes worse. In the case of $Q=1$, the PDS algorithm is used again at step 3, now with $M_{b}=1$, i.e., the second PDS algorithm provides the bitflipping, which results in significant improvement in the BER performance. For the highly loaded scenario, the performance becomes very close to the single-user bound. We compare the 


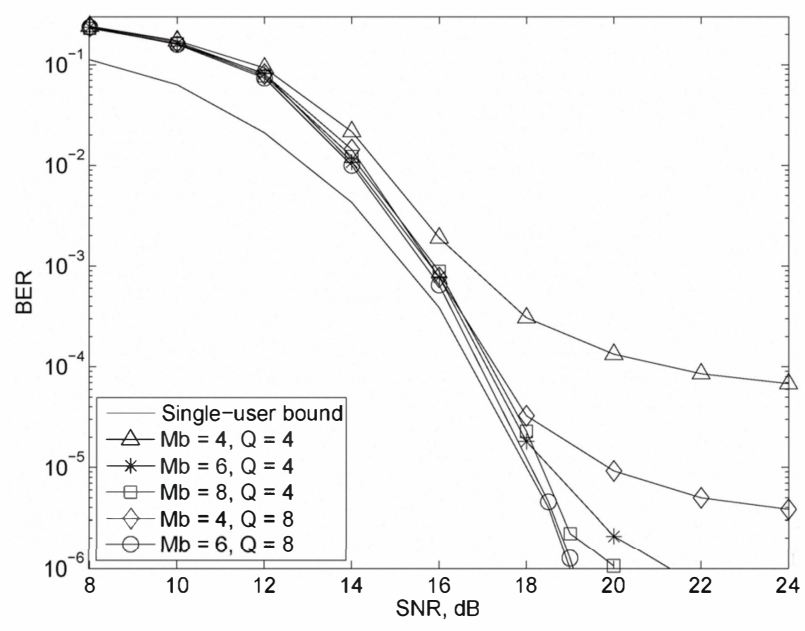

Fig. 2. BER performance of the multiple phase detector; 8-PSK modulation, $K=60, S F=63$.

performance with the single-user bound (instead of the ML performance) because simulation of the ML detector with $K=60$ users would be impractical.

Fig. 2 shows the BER performance for a scenario with 8-PSK modulation. For this scenario, the use of $Q=0$ or $Q=1$ (not shown here) does not allow the detection performance to approach the single-user bound. For $Q=4$, as $M_{b}$ increases, the BER floor level is reduced, whereas the BER curves slightly depart from the single-user bound. However, for $Q=8$ and $M_{b}=6$ at high SNRs, the BER performance of the proposed detector is very close to the single-user bound.

In the simulation, we computed the average number of multiplications in the proposed detector with the best performance in Fig.2, i.e., $Q=8$ and $M_{b}=6$. On average, the detector requires approximately $5 \cdot 10^{5}$ multiplications to detect all $K=60$ user symbols. Note that the complexity of the decorrelator (one of the simplest detectors) is approximately $K^{3} \approx 2 \cdot 10^{5} \mathrm{mul}-$ tiplications, i.e., the complexity of the proposed multiple phase detector with $Q=8$ branches in such a highly loaded scenario is close to that of the decorrelator. In [4], complexity results for the SDR detector are presented; specifically, for $K=10$, the SDR detector requires approximately $2 \cdot 10^{6}$ multiplications. Our simulation results have shown that, in the case of $K=10$, $Q=8$, and $M_{b}=6$, the proposed detector requires on average $1.2 \cdot 10^{4}$ multiplications, i.e., two orders of magnitude lower than that of the SDR detector.

Fig. 3 compares the symbol-error-rate (SER) performance of the proposed detector against the SDR detector for 8-PSK modulation. The results for the SDR detector are taken from [4], where the simulation has been done with Gold signature waveforms of length $\mathrm{SF}=31$. We also use the Gold signature waveforms in the same scenarios, where the number of users varies from $K=20$ to $K=30$. Fig. 3 shows that the proposed detector significantly outperforms the SDR detector and the performance of our detector is very close to the single-user bound. We have also repeated the simulation for binary signature waveforms randomly chosen in each simulation trial; it can be seen that the performance is similar or better than that of the SDR detector with Gold signature waveforms.

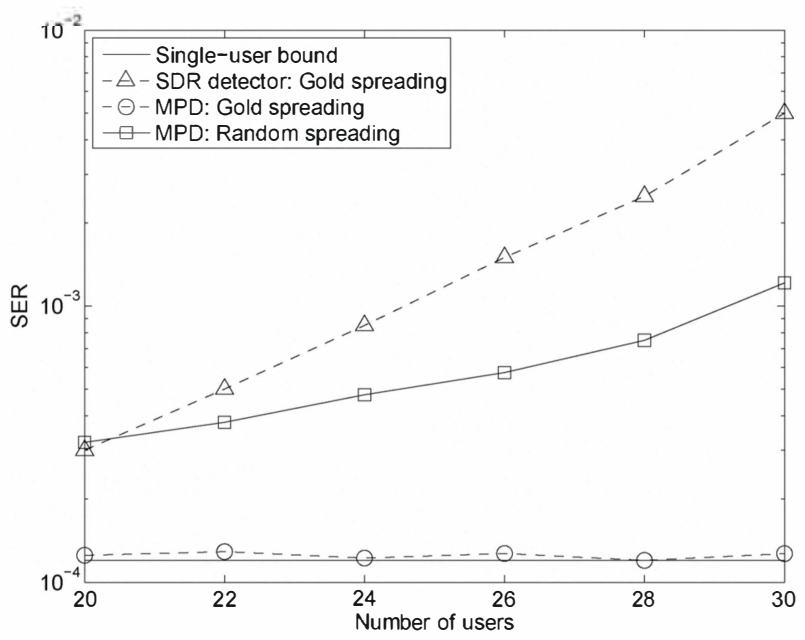

Fig. 3. SER performance of the multiple phase detector against the SDR detector; 8-PSK modulation, $Q=4, M_{b}=8, \mathrm{SF}=31$, $\mathrm{SNR}=17 \mathrm{~dB}$.

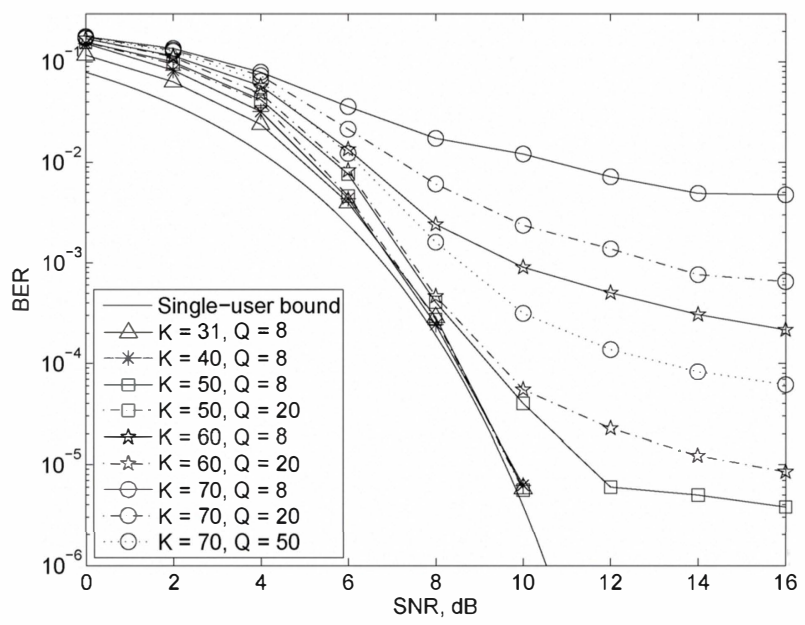

Fig. 4. BER performance of the multiple phase detector in overloaded scenarios; BPSK modulation, $\mathrm{SF}=31$.

Fig. 4 shows the BER performance of the proposed detector in overloaded multiuser scenarios with BPSK modulation where the number of users $K$ exceeds the spreading factor SF. The signature waveforms are random binary with equal energies. It is seen that the proposed detector allows reliable detection even in these difficult situations. E.g., for $K=60$ and as high a load as $K / \mathrm{SF} \approx 2$, at $\mathrm{BER}=10^{-4}$ the detection performance departs from the single-user bound by as little as approximately $1 \mathrm{~dB}$. However, this requires an increase in the number of branches (initializations) $Q$.

Finally, we provide simulation results that demonstrate nearfar resistance of the proposed detector. We consider scenarios where all but the first user has the same SNR [5]. In Fig.5, the BER of the first user is shown against the ratio of the strength of the interfering user signals to the first user signal for BPSK modulation. In the case of $K=10$ and $\mathrm{SF}=32$ (this case and the case $K=24$ and $\mathrm{SF}=32$ are similar to that considered in [5]), the near-far resistance of the proposed detector is close to that of the ML detector. In this case, increase in $Q$ does not improve the BER performance. In the case of $K=24$, in- 


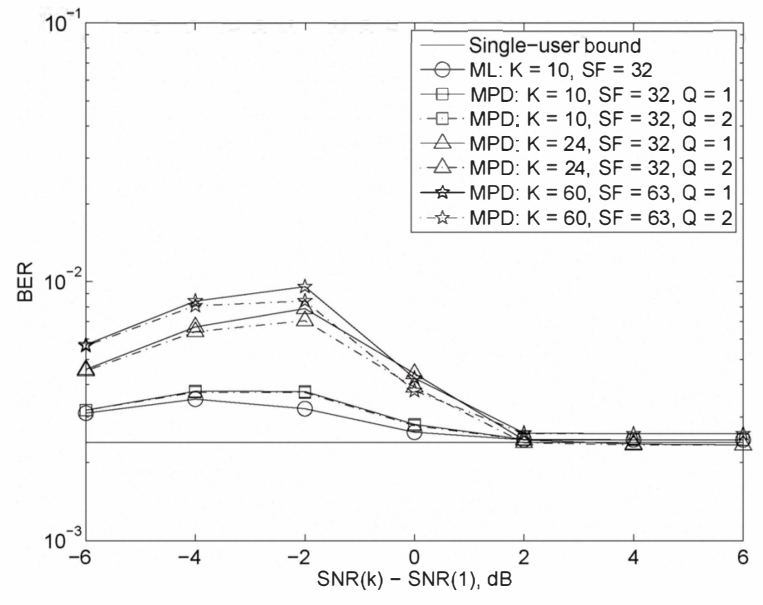

Fig. 5. BER performance of the multiple phase detector in near-far scenarios; BPSK modulation, $\mathrm{SNR}(1)=6 \mathrm{~dB}$.

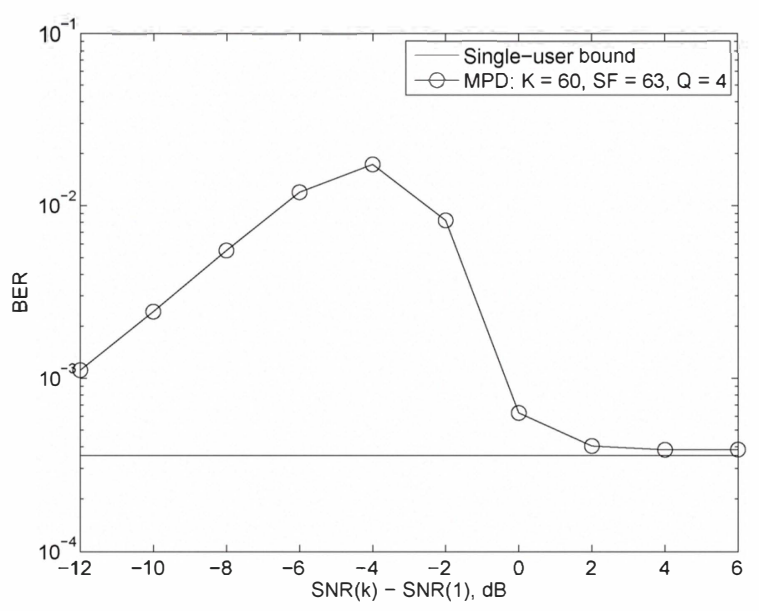

Fig. 6. BER performance of the multiple phase detector in near-far scenarios; 8-PSK modulation, $\mathrm{SNR}(1)=16 \mathrm{~dB}$.

crease in $Q$ results in a slight improvement. Note that the direct simulation of the ML detector for $K=24$ is impractical, therefore we cannot compare our results with the ML performance. In [5], the BER performance of an ML detector implemented using a branch and bound algorithm based on SDR is given for the same scenario. However, our results show slightly better near-far resistance compared to the ML performance presented in [5] ${ }^{1}$. It is seen that with further increase of the load (the case of $K=60$ and $\mathrm{SF}=63$ ), the proposed detector demonstrates small variations in the near-far resistance. Fig. 6 shows that the proposed detector has also good near-far resistance for 8-PSK modulation.

\section{CONCLUSIONS}

We have used a novel iterative technique, the phase descent search algorithm, for joint detection of multiple M-PSK symbols, specifically for multiuser detection. The technique provides an approximate solution to the quadratic optimization problem with a constraint that forces elements of the solution to

\footnotetext{
${ }^{1}$ This could be due to approximations made in [5] or/and a smaller number of simulation trials used in [5]
}

have unit magnitude. This technique is used multiple times in the multiuser detector. Simulation results show that for highly loaded scenarios this technique has detection performance that is close to the single-user bound. The technique also allows reliable detection in highly overloaded scenarios and demonstrates good near-far resistance. We have shown by simulation that the multiple phase detector has a performance that is significantly better, and complexity that is significantly lower, than that of the detector based on semi-definite relaxation.

\section{REFERENCES}

[1] S. Verdu, Multiuser Detection, Cambridge University Press, 1998.

[2] E. Agrell, T. Eriksson, A. Vardy, and K. Zeger, "Closest point search in lattices," IEEE Trans. Inf. Theory, vol. 48, no. 8, pp. 2201-2214, Aug. 2002.

[3] J. Jalden and B. Otterson, "On the complexity of sphere decoding in digital communications," IEEE Trans. Signal Processing, vol. 53, no. 4, pp. 1474-1484, Apr. 2005.

[4] W.-K. Ma, P.-C. Ching, and Z. Ding, "Semidefinite relaxation based multiuser detection for M-ary PSK multiuser systems," IEEE Trans. Signal Processing, vol. 52, no. 10, pp. 2862-2872, Oct. 2004.

[5] P. H. Tan and L. K. Rasmussen, "Multiuser detection in CDMA - A comparison of relaxations, exact, and heuristic search methods," IEEE Trans. Wireless Commun., vol. 3, no. 5, pp. 1802-1809, Sep. 2004.

[6] Y. V. Zakharov and T. C. Tozer, "Multiplication-free iterative algorithm for LS problem," Electronics Letters, vol. 40, no. 9, pp. 567-569, April 2004.

[7] Y. V. Zakharov and T. C. Tozer, "Box-constrained multiuser detection based on multiplication-free coordinate descent optimisation," in Proceedings of the V IEEE Signal Processing Workshop on "Signal Processing Advances in Wireless Communications", 11-14 July 2004, Lisbon.

[8] Z. Quan, J. Liu, and Y. Zakharov, "FPGA implementation of DCD based CDMA multiuser detector," 15th International Conference on Digital Signal Processing, 2007, Cardiff, UK, pp. 319-322, 2007.

[9] Z. Quan, Y. Zakharov, and J. Zhang, "Multiple phase decoder for MIMO systems," in Proc. 42 Asilomar Conf. Signals, Systems, and Computers, Pacific Grove, CA, US, Oct 2008, pp. 1759 - 1762.

[10] F. P. Vasiliev, Numerical methods for solution of extremum problems (in Russian), Nauka, Moscow, 1988. 